\title{
Optimal operation of a three-level quantum heat engine and universal nature of efficiency
}

\author{
Varinder Singh (๑* \\ Department of Physics, Koç University, Sarlyer, Istanbul 34450, Turkey
}

(Received 6 April 2020; accepted 19 October 2020; published 5 November 2020)

\begin{abstract}
We present a detailed study of a three-level quantum heat engine operating at maximum efficient power function, a trade-off objective function defined by the product of the efficiency and power output of the engine. The efficient power function represents a trade-off between the power output and efficiency of a heat engine. Engines working in the maximum efficient power regime operate at finite power, with finite efficiency lying in between the regimes of maximum power and maximum efficiency (Carnot efficiency). First, for near-equilibrium conditions, we find a general expression for the efficiency and establish the universal nature of efficiency at maximum power and maximum efficient power. Then in the high-temperature limit, optimizing with respect to one parameter while constraining the other one, we obtain the lower and upper bounds on the efficiency for both strong as well as weak matter-field coupling conditions. Except for the weak matter-field coupling condition, the obtained bounds on the efficiency exactly match with the bounds already known for some models of classical heat engines. Further for weak matter-field coupling, we derive some new bounds on the efficiency of the engine which lie beyond the range covered by bounds obtained for strong matter-field coupling. We conclude by comparing the performance of our three-level quantum heat engine in maximum power and maximum efficient power regimes and show that the engine operating at maximum efficient power produces at least $88.89 \%$ of the maximum power output while considerably reducing the power loss due to entropy production. Finally, to complete our analysis, we review the experimental setup of a recently realized effective three-level quantum engine.
\end{abstract}

DOI: 10.1103/PhysRevResearch.2.043187

\section{INTRODUCTION}

The study of quantum heat engines (QHEs) started with the seminal work of Scovil and Schulz-DuBois (SSD) [1]. In their work, they investigated the thermodynamics of a three-level maser and showed that its limiting efficiency is given by Carnot efficiency [2]. Since then, three-level systems have been employed to study various models of quantum heat engines (refrigerators) [3-27] and quantum absorption refrigerators [28-36].

Here, we specifically mention the work of Geva and Kosloff [4-6] on three-level amplifier. They studied the SSD engine in the spirit of finite-time thermodynamics using Alicki's definition of heat and work [37], and optimized its performance with respect to different control parameters. They showed that in the presence of external electromagnetic field, one has to incorporate the effect of the field on the dissipation superoperators in order to satisfy the second law of thermodynamics. Going one step further, Tannor and Boukobza formulated a new way of partitioning energy into heat and work [10-12]. They applied their formulation to a three-level system simultaneously coupled to two thermal

\footnotetext{
*vsingh@ku.edu.tr

Published by the American Physical Society under the terms of the Creative Commons Attribution 4.0 International license. Further distribution of this work must maintain attribution to the author(s) and the published article's title, journal citation, and DOI.
}

baths at different temperatures and to a single-mode of classical electromagnetic field, and showed that the second law of thermodynamics is always satisfied without incorporating the effect of the field on the dissipators [12]. Recently, their formalism has been used to study the phenomenon of noiseinduced coherence [23] and quantum synchronization [38] in nanoscale engines.

In this work, we use Tannor and Boukobza's formalism to analyze the optimal performance of the SSD engine and set up its correspondence with some classical models of heat engines. At optimal performance, QHEs operating at finite power, show remarkable similarity to classical macroscopic heat engines. For instance, in high-temperature limit, many models of QHEs [23,39-44] operate at CurzonAhlborn (CA) efficiency, a well-known result in the field of finite-time thermodynamics [45-47], first obtained for a macroscopic model of heat engine known as endoreversible engine [48,49]. Similarly, in the low-dissipation regime [50], the behavior of quantum and classical heat engines are quite similar [51,52].

One another feature common in the operation of classical and QHEs is the universal nature of efficiency [53]. Many models of classical and QHEs show the universality of efficiency at maximum power (EMP) upto quadratic order in $\eta_{C}$, i.e., $\eta_{\mathrm{MP}}=\eta_{C} / 2+\eta_{C}^{2} / 8+\mathcal{O}\left(\eta_{C}^{3}\right)$. Van den Broeck proved that in the linear response regime, $\eta_{C} / 2$ is universal for tight-coupling heat engines [54]. Further, Esposito and coauthors established the universality of the second term $\eta_{C}^{2} / 8$ by invoking the symmetry of Onsager coefficients on the nonlinear level [53]. 
The universal features of efficiency are not unique to the EMP, two other optimization functions: omega $(\Omega)$ function (or ecological function) $[55,56]$ and efficient power (EP) function [57,58], $P_{\eta}=\eta P$ (product of the efficiency and power of the engine), also exhibit universal behavior $[59,60]$. Here, we will discuss the universal character of efficiency at maximum efficient power (EMEP) only. The formal proof of the universality of EMEP was established in Ref. [60]. It was shown that the first two universal terms are $2 \eta_{C} / 3$ and $2 \eta_{C}^{2} / 27$.

In this paper, we study the optimal performance of the SSD engine operating at maximum efficient power (MEP), a tradeoff optimization function representing a trade-off between the power output and efficiency of a heat engine, in different operational regimes and compare its performance with the engine operating at maximum power (MP). The study of such objective functions is important from the environmental and ecological point of view. It is already known that engines operating in the MP regime also waste a lot of power due to large entropy production $[61,62]$. Therefore, rather than operating in the MP regime, the real heat engines should operate near the MP regime, where they produce slightly smaller power output with appreciable larger efficiency, which makes them costeffective too [62]. The EP function was introduced by Stucki [57] in the context of the biochemical energy conversion process. Engines working in the maximum efficient power regime operate at finite power, with finite efficiency lying in between the regimes of maximum efficiency (or Carnot efficiency) and maximum power. Extending Stucki's idea, Yan and Chen (YC) treated EP function as their objective function to investigate the performance of an endoreversible heat engine [58]. Recently, EP function has attracted considerable interest and have been employed to study the energy conversion process in low-dissipation heat engines $[63,64]$, thermionic generators [65], biological systems [57,66], chemical reactions [67,68], Feynman's ratchet and pawl model [69], and in a quantum Otto engine [70].

The paper is organized as follows. In Sec. II, we discuss the model of the SSD engine. In Sec. III, we obtain an analytic expression for the efficiency of the the SSD engine operating near-equilibrium conditions and show the universality of the EMP and EMEP. In Secs. IV A and IV B, we optimize engine's performance, operating in two different operational regimes (strong and weak matter-field coupling regimes), with respect to one parameter only, and obtain the lower and upper bounds on the EMEP for each case. Section IV C is devoted to the discussion of universality of efficiency for one parameter optimization scheme under the effect of some symmetric constraints imposed on the control parameters of the engine. In Secs. V and VI, we compare the performance of the SSD engine operating at MEP to the engine operating at MP. In Sec. VII, we discuss the experimental setup of a recently realized effective three-level quantum engine. We conclude in Sec. VIII.

\section{MODEL OF A THREE-LEVEL QUANTUM LASER HEAT ENGINE}

SSD engine [1] is one of the simplest QHEs. Using the concept of stimulated emission in a population inverted medium, it converts the incoherent thermal energy of heat reservoirs to
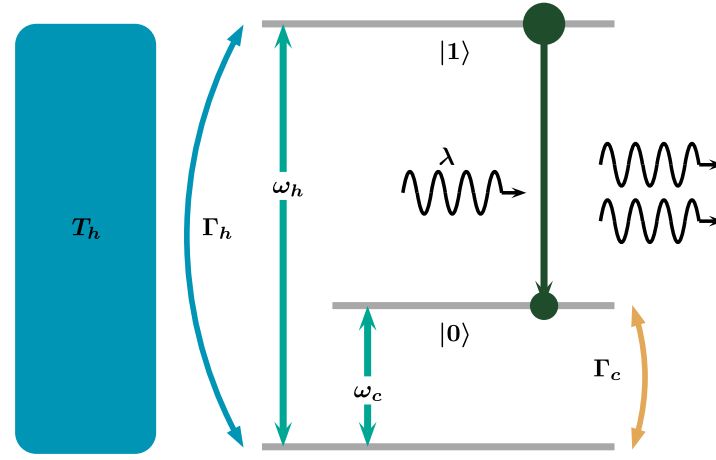

$|g\rangle$

FIG. 1. Model of the SSD engine simultaneously coupled to two thermal reservoirs at temperatures $T_{c}$ and $T_{h}$ with coupling constants $\Gamma_{c} \Gamma_{h}$, respectively. The interaction of the system with a classical single-mode field is represented by $\lambda$, the matter-field coupling constant.

a coherent laser output. The model consists of a three-level system simultaneously coupled to two thermal reservoirs at temperatures $T_{h}$ and $T_{c}\left(T_{c}<T_{h}\right)$, and to a single-mode classical electromagnetic field (see Fig. 1). The hot reservoir at temperature $T_{h}$ induces the transition between the ground state $|g\rangle$ and the upper state $|1\rangle$, whereas the transition between the middle state $|0\rangle$ and the ground state $|g\rangle$ is constantly de-excited by the cold reservoir at temperature $T_{c}$. For the power output mechanism, states $|0\rangle$ and $|1\rangle$ are coupled to a classical single-mode field. The bare Hamiltonian of the threelevel system is given by: $H_{0}=\hbar \sum \omega_{k}|k\rangle\langle k|$ where the sum runs over all three states and $\omega_{k}$ 's represent the corresponding atomic frequencies. Under the rotating wave approximation, the following semiclassical Hamiltonian describes the interaction of the system with the classical field of frequency $\omega: V(t)=\hbar \lambda\left(e^{-i \omega t}|1\rangle\left\langle 0\left|+e^{i \omega t}\right| 0\right\rangle\langle 1|\right) ; \lambda$ is the field-matter coupling constant. The reduced dynamics of the matter-field system under the effect of the heat reservoirs is described by the following form of Lindblad master equation:

$$
\dot{\rho}=-\frac{i}{\hbar}\left[H_{0}+V(t), \rho\right]+\mathcal{L}_{h}[\rho]+\mathcal{L}_{c}[\rho],
$$

where $\mathcal{L}_{h}$ and $\mathcal{L}_{c}$ are the dissipation Lindblad superoperators describing the interaction of the system with the hot and cold reservoirs, respectively:

$$
\begin{aligned}
\mathcal{L}_{h}[\rho]= & \Gamma_{h}\left(n_{h}+1\right)\left(2|g\rangle\left\langle g\left|\rho_{11}-\right| 1\right\rangle\langle 1|\rho-\rho| 1\rangle\langle 1|\right) \\
& +\Gamma_{h} n_{h}\left(2|1\rangle\left\langle 1\left|\rho_{g g}-\right| g\right\rangle\langle g|\rho-\rho| g\rangle\langle g|\right), \\
\mathcal{L}_{c}[\rho]= & \Gamma_{c}\left(n_{c}+1\right)\left(2|g\rangle\left\langle g\left|\rho_{00}-\right| 0\right\rangle\langle 0|\rho-\rho| 0\rangle\langle 0|\right) \\
& +\Gamma_{c} n_{c}\left(2|0\rangle\left\langle 0\left|\rho_{g g}-\right| g\right\rangle\langle g|\rho-\rho| g\rangle\langle g|\right),
\end{aligned}
$$

where $\Gamma_{c}$ and $\Gamma_{h}$ are Weisskopf-Wigner decay constants, and $n_{h(c)}=1 /\left(\exp \left[\hbar \omega_{h(c)} / k_{B} T_{h(c)}\right]-1\right)$ is average number of photons in the mode of frequency $\omega_{h(c)}$ in hot (cold) reservoir satisfying the relations $\omega_{c}=\omega_{0}-\omega_{g}, \omega_{h}=\omega_{1}-\omega_{g}$.

To solve the density matrix equations, it is convenient to transform to a rotating frame in which semiclassical interaction Hamiltonian and the steady-state density matrix $\rho_{R}$ 
becomes time-independent [12]. Defining $\bar{H}=\hbar\left(\omega_{g}|g\rangle\langle g|+\right.$ $\left.\frac{\omega}{2}|1\rangle\left\langle 1\left|-\frac{\omega}{2}\right| 0\right\rangle\langle 0|\right)$, an arbitrary operator $B$ in the rotating frame is given by $B_{R}=e^{i \bar{H} t / \hbar} B e^{-i \bar{H} t / \hbar}$. It can be seen that superoperators $\mathcal{L}_{c}[\rho]$ and $\mathcal{L}_{h}[\rho]$ remain unchanged under this transformation. Finally, the time evolution of the system density operator in this rotating frame is given by

$$
\dot{\rho_{R}}=-\frac{i}{\hbar}\left[H_{0}-\bar{H}+V_{R}, \rho_{R}\right]+\mathcal{L}_{h}\left[\rho_{R}\right]+\mathcal{L}_{c}\left[\rho_{R}\right]
$$

where $V_{R}=\hbar \lambda(|1\rangle\langle 0|+| 0\rangle\langle 1|)$.

For a weak system-bath coupling, the heat flux, output power, and efficiency of the SSD engine can be defined, using the formalism of Ref. [12], as follows:

$$
\begin{gathered}
\dot{Q}_{h}=\operatorname{Tr}\left(\mathcal{L}_{h}\left[\rho_{R}\right] H_{0}\right), \\
P=\frac{i}{\hbar} \operatorname{Tr}\left(\left[H_{0}, V_{R}\right] \rho_{R}\right), \\
\eta=\frac{P}{\dot{Q}_{h}} .
\end{gathered}
$$

Here, we have used the sign convention in which all three energy fluxes: heat flux extracted from the hot bath, heat flux rejected to the cold bath and the power output are positive. Substituting the expressions for $V_{R}, H_{0}$, and $\mathcal{L}_{h}\left[\rho_{R}\right]$, and calculating the traces appearing in Eqs. (5) and (6) (see Appendix A), the heat flux and power output can be written as

$$
\begin{gathered}
\dot{Q}_{h}=i \hbar \lambda \omega_{h}\left(\rho_{01}-\rho_{10}\right), \\
P=i \hbar \lambda\left(\omega_{1}-\omega_{0}\right)\left(\rho_{01}-\rho_{10}\right), \\
=i \hbar \lambda\left(\omega_{h}-\omega_{c}\right)\left(\rho_{01}-\rho_{10}\right),
\end{gathered}
$$

where $\rho_{01}=\left\langle 0\left|\rho_{R}\right| 1\right\rangle$ and $\rho_{10}=\left\langle 1\left|\rho_{R}\right| 0\right\rangle$. Using Eqs. (8) and (9) in Eq. (7), the efficiency of the engine is given by

$$
\eta=1-\frac{\omega_{c}}{\omega_{h}} .
$$

The positive power production condition [see Eq. (A11)] implies that $\omega_{c} / \omega_{h} \geqslant T_{c} / T_{h}$, which in turn implies that $\eta \leqslant \eta_{C}$.

\section{UNIVERSAL NATURE OF THE EFFICIENCY}

In this section, we will explicitly show the universal nature of both EMP and EMEP. The expressions for the power output and EP are derived in Appendix A [Eqs. (A11) and (A12)] Optimization of these equations with respect to control parameters $\omega_{h}$ and $\omega_{c}$ yields very complex equations, which cannot be solved analytically under the general conditions. However, close to the equilibrium, they can be solved to yield an analytic expression for the efficiency upto the second-order term in $\eta_{C}$, which is sufficient for our purpose as we want to focus only on the universal nature of the EMP and EMEP.

As mentioned in Introduction, the appearance of the first two universal terms in the Taylor series of the EMP was first proven by Esposito and coauthors for tight-coupling heat engines possessing a left-right symmetry in the system. We briefly outline the algorithm followed in Ref. [53]. The following formalism is valid for the engines obeying tightcoupling condition between the energy flux $I_{E}$ and matter flux $I$ :

$$
I_{E}(x, y)=\epsilon I(x, y),
$$

where $x$ and $y$ are dimensionless scaled energies (to be specified later). The above equation implies that the energy is exported by the particles of a given energy $\epsilon$. The general formula for the EMP is given by

$$
\eta=\frac{\eta_{C}}{2}+\left(1+\frac{M}{\partial_{x} L}\right) \frac{\eta_{C}^{2}}{4}+\mathcal{O}\left(\eta_{C}^{3}\right)
$$

where $L=-I_{1}^{\prime}(x, x)$ and $M=I_{11}^{\prime \prime}(x, x) / 2$. Further, for the systems possessing a left-right symmetry, the inversion of flux,

$$
I(x, y)=-I(y, x)
$$

leads to the condition $2 M=-\partial_{x} L$, which reduces the second term in Eq. (12) to $\eta_{C}^{2} / 8$, thus establishing the universality of the coefficient $1 / 8$ under the symmetry specified by Eq. (13).

To explore the universal nature of the efficiency in the SSD model, we must first identify the flux term. In our model, energy is transported from hot to cold reservoir by the flux of photons. Comparing Eq. (A11) with Eq. (11), we identify $I$ as follows:

$$
I(x, y)=\frac{2 \lambda^{2} \Gamma_{c} \Gamma_{h}\left(e^{x}-e^{y}\right)}{\lambda^{2}\left[\left(e^{x}+2\right)\left(e^{y}-1\right) \Gamma_{c}+\left(e^{x}-1\right)\left(e^{y}+2\right) \Gamma_{h}\right]+\Gamma_{c} \Gamma_{h}\left(e^{x+y}+e^{x}+e^{y}\right)\left(\frac{e^{x} \Gamma_{c}}{e^{x}-1}+\frac{e^{y} \Gamma_{h}}{e^{y}-1}\right)} .
$$

In the above equation, we have put $x \equiv \hbar \omega_{c} / k_{B} T_{c}$ and $y \equiv \hbar \omega_{h} / k_{B} T_{h}$, and used the expressions $n_{h}=1 /\left(e^{y}-1\right)$ and $n_{c}=1 /\left(e^{x}-1\right)$. By inspecting Eq. (14), we can see that symmetry criterion (13) is satisfied for $\Gamma_{h}=\Gamma_{c}$. Under this condition, we should observe the universality of efficiency for the SSD model. We confirm this observation by explicitly calculating the form of efficiency in Eq. (12). By evaluating expressions for $L, M$, and $\partial_{x} L$ for the current $I$ given in Eq. (14), and substituting in Eq. (12), we find

$$
\eta=\frac{\eta_{C}}{2}+\frac{\eta_{C}^{2}}{4}\left[\frac{\left(\left(e^{\alpha}+1\right) \Gamma_{c}\left(\left(e^{\alpha}-1\right)^{2}\left(e^{\alpha}+2\right) \lambda^{2}+e^{2 \alpha}\left(e^{\alpha}-4\right) \Gamma_{h}^{2}\right)+e^{3 \alpha}\left(e^{\alpha}-1\right) \Gamma_{c}^{2} \Gamma_{h}+\left(e^{\alpha}-2\right)\left(e^{\alpha}-1\right)^{3} \lambda^{2} \Gamma_{h}\right)}{2\left(\Gamma_{c}+\Gamma_{h}\right)\left(\left(e^{\alpha}-1\right)^{2}\left(e^{2 \alpha}+2\right) \lambda^{2}+e^{2 \alpha}\left(e^{\alpha}\left(e^{\alpha}-2\right)-2\right) \Gamma_{c} \Gamma_{h}\right)}\right]+\mathcal{O}\left(\eta_{C}^{3}\right),
$$


where $\alpha$ is the solution of the transcendental equation, ${ }^{1}$

$$
\alpha\left(-\frac{1}{e^{\alpha}+2}+\frac{1}{2-2 e^{\alpha}}+\frac{\left(e^{\alpha}-1\right) \lambda^{2}}{\left(e^{\alpha}-1\right)^{2} \lambda^{2}+e^{2 \alpha} \Gamma_{c} \Gamma_{h}}+\frac{1}{2}\right)=1,
$$

which can be solved by specifying the numerical values of $\lambda$, $\Gamma_{h}$ and $\Gamma_{c}$. For $\lambda^{2}=\Gamma_{h} \Gamma_{c}$, the solution of the above equation yields $\alpha=2.9327$. For the symmetric dissipation, $\Gamma_{c}=\Gamma_{h}$, the term inside the square bracket in Eq. (15) becomes equal to $1 / 2$, yielding the coefficient of the second term as $1 / 8$, and, hence proving our assertion.

\section{A. Universality of the EMEP}

The general expression for the EMEP analogous to Eq. (12) is [60]

$$
\eta=\frac{2 \eta_{C}}{3}+\left(1+\frac{M}{\partial_{x} L}\right) \frac{4 \eta_{C}^{2}}{27}+\mathcal{O}\left(\eta_{C}^{3}\right)
$$

Comparing Eqs. (12) and (17), we can see that in order to obtain the explicit expression for the EMEP, we just have to replace $\eta_{C}^{2} / 4$ by $4 \eta_{C}^{2} / 27$ in Eq. (15); everything else remains the same. Since, for $\Gamma_{h}=\Gamma_{c}$, the term inside the square bracket in Eq. (15) is $1 / 2$, we obtain the second term as $2 \eta_{C}^{2} / 27$.

From the above procedure, we can conclude that if the universal nature of the EMP can be established for a model under consideration, the universality of the EMEP automatically follows. The universal character of the EMEP has already been established for the low-dissipation [63], endoreversible [58,71], and nonlinear irreversible [60] models of classical heat engine. Ours is the first study of a QHE in which the universality of the EMEP is explored and explicitly shown.

\section{B. Global optimization of efficient power function in the low-temperature limit}

Now, we study the operation of the SSD engine in the low-temperature regime. In the low-temperature limit, we assume $\hbar \omega_{c, h} \gg k_{B} T_{c, h}$, such that $n_{c, h} \simeq e^{-\hbar \omega_{c, h} / k_{B} T_{c, h}} \ll 1$. The EP function [Eq. (A12)] in this case is given by

$$
P_{\eta}=\frac{2 \hbar \lambda^{2} \Gamma_{c} \Gamma_{h}\left(n_{h}-n_{c}\right)\left(\omega_{h}-\omega_{c}\right)^{2}}{\omega_{h}\left(\Gamma_{c}+\Gamma_{h}\right)\left(\lambda^{2}+\Gamma_{c} \Gamma_{h}\right)} .
$$

In our previous work [25], we have proven the equivalence of the SSD engine operating in the low-temperature limit to Feynman's ratchet and pawl engine [72-74], a classical heat engine based on the principle of Brownian fluctuations. Hence, the analysis of this section is also valid for Feynman's model. Maximization of Eq. (18) with respect to $\omega_{h}$ and $\omega_{c}$, and a little simplification yields the following equations:

$$
\begin{aligned}
e^{\hbar \omega_{h} / k_{B} T_{h}-\hbar \omega_{c} / k_{B} T_{c}} & =1-\frac{\hbar \omega_{h}\left(\omega_{h}-\omega_{c}\right)}{k_{B} T_{h}\left(\omega_{h}+\omega_{c}\right)}, \\
e^{\hbar \omega_{h} / k_{B} T_{h}-\hbar \omega_{c} / k_{B} T_{c}} & =\frac{2 k_{B} T_{c}}{\hbar\left(\omega_{h}-\omega_{c}\right)+2 k_{B} T_{c}} .
\end{aligned}
$$

\footnotetext{
${ }^{1}$ The transcendental equation is obtained by substituting the expression for $L$ and $\partial_{x} L$ in equation $x=-2 L / \partial_{x} L$ (see Ref. [53]).
}

It is not possible to obtain an analytic solution of these two equations for $\omega_{h}$ and $\omega_{c}$. However, combining Eqs. (10), (19) and (20), and writing in terms of $\eta_{C}=1-T_{c} / T_{h}$, we obtain the following transcendental equation:

$$
\frac{\left(2 \eta_{C}-\eta\right)\left(\eta-\eta_{C}\right)}{\eta\left(1-\eta_{C}\right)}=\ln \left[\frac{2\left(1-\eta_{C}\right)}{2-\eta}\right] \text {. }
$$

It is clear from the Eq. (21) that the efficiency does not depend on the system-parameters and depends on $\eta_{C}$ only. We plot Eq. (21) in Fig. 2 and compare the EMEP (EMP) of the cold the SSD engine with the corresponding EMEP (EMP) of endoreversible or low-dissipation heat engines.

Near equilibrium, a perturbative solution for the Eq. (21) is available and is given by [69]

$$
\eta_{\text {cold }}^{S S D}=\frac{2 \eta_{C}}{3}+\frac{2 \eta_{C}^{2}}{27}+\frac{11 \eta_{C}^{3}}{243}+\mathcal{O}\left(\eta_{C}^{4}\right) .
$$

Hence, again in this regime, we are able to show the existence of the first two universal terms $\left(2 \eta_{C} / 3\right.$ and $\left.2 \eta_{C}^{2} / 27\right)$ for a twoparameter optimization scheme.

\section{LOCAL OPTIMIZATION}

Since for the unconstrained regime, general solution for the two-parameter optimization of the SSD engine is not available, in the following, we will optimize the performance of the engine with respect to one control parameter only while

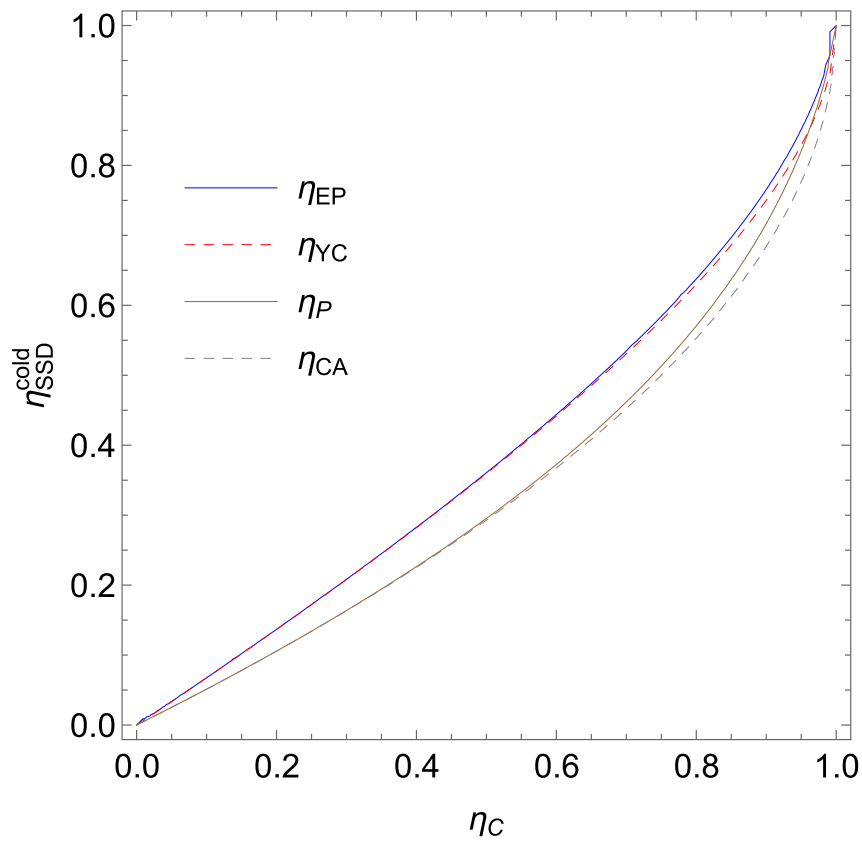

FIG. 2. Comparison of the EMEP (EMP) of the SSD engine operating in low-temperature limit with $\eta_{Y C}\left(\eta_{C A}\right)$. The EMEP (EMP) of the SSD engine is higher than that of low-dissipation and endoreversible engines which operate at $\eta_{Y C}\left(\eta_{C A}\right)$. 
keeping the other one fixed at a constant value. In the hightemperature limit, it is possible to obtain analytic expressions for the EMP and EMEP. In this limit, we put $n_{h} \approx k_{B} T_{h} / \hbar \omega_{h}$ and $n_{c} \approx k_{B} T_{c} / \hbar \omega_{c}$.

\section{A. High-temperature and strong-coupling regime}

Assuming the strong matter-field coupling $\left(\lambda \gg \Gamma_{h, c}\right)$, the expression for EP function in the high-temperature limit can be written as

$$
P_{\eta}=\frac{2 \hbar \Gamma_{h}\left(\omega_{h}-\omega_{c}\right)^{2}\left(\omega_{c}-\tau \omega_{h}\right)}{3 \omega_{c}\left(\omega_{c} \gamma+\tau \omega_{h}\right)} .
$$

It is important to mention that two-parameter optimization of the EP function given in Eq. (23) is not possible. Such a two-parameter optimization scheme leads to the trivial solution, $\omega_{c}=\omega_{h}=0$, which is not useful result. It indicates that a unique maximum of $P_{\eta}$ with respect to both $\omega_{c}$ and $\omega_{h}$ cannot exist. It can be argued as follows. For the given values of the bath temperatures and the coupling constants, under the scaling $\left(\omega_{c}, \omega_{h}\right) \rightarrow\left(\beta \omega_{c}, \beta \omega_{h}\right)$, where $\beta$ is a certain positive number, the EP function also scales as $P_{\eta} \rightarrow \beta P_{\eta}$. Hence, there cannot exist a unique optimal solution $\left(\omega_{h}^{*}, \omega_{c}^{*}\right)$ that yields a unique maximum for EP function. The same is also true for the optimization of the power output of the engine with respect to $\omega_{c}$ and $\omega_{h}$. Therefore we will perform optimization with respect to one parameter only while keeping the other one fixed. First, we keep $\omega_{h}$ fixed, then optimizing EP [Eq. (23)] with respect to $\omega_{c}$, i.e., setting $\partial P_{\eta} / \partial \omega_{c}=0$, we evaluate EMEP as

$$
\eta_{\omega_{h}}^{P \eta}=1-\frac{(\gamma-3) \tau+\sqrt{\gamma+1} \sqrt{\tau} \sqrt{\gamma(\tau+8)+9 \tau}}{4 \gamma},
$$

where $\gamma=\Gamma_{h} / \Gamma_{c}$. For a given value of $\tau, \eta_{\omega_{h}}^{P \eta}$ is a monotonically increasing function of $\gamma$. Hence, we can obtain the lower and upper bounds of EMEP by setting $\gamma \rightarrow 0$ and $\gamma \rightarrow \infty$, respectively. Writing in terms of $\eta_{C}=1-\tau$, we have

$$
\frac{2 \eta_{C}}{3} \leqslant \eta_{\omega_{h}}^{P \eta} \leqslant 1-\frac{1}{4}\left(1-\eta_{C}\right)\left(1+\sqrt{1+\frac{8}{1-\eta_{C}}}\right) \equiv \eta_{Y C} .
$$

Recently, $\eta_{-}^{P_{\eta}} \equiv 2 \eta_{C} / 3$, has also been obtained as the lower bound of the low-dissipation heat engines operating at MEP $[63,64]$. The upper bound $\eta_{Y C}$ obtained here was first obtained by Yan and Chen for an endoreversible heat engine [58]. Hence, we name it after them. $\eta_{Y C}$ can also be obtained for symmetric low-dissipation heat engines [63].

Alternately, we may fix the value of $\omega_{c}$ and optimize EP function with respect to $\omega_{h}$. In this case, we get the following equation:

$$
\frac{\tau^{2} \omega_{h}^{3}+\tau(2 \gamma+\tau) \omega_{c} \omega_{h}^{2}-(\gamma+2 \tau) \omega_{c}^{2} \omega_{h}-\gamma \omega_{c}^{3}}{\omega_{c} \gamma+\omega_{h} \tau}=0 .
$$

Due to Casus irreducibilis (see Appendix C), the roots of the cubic polynomial in the numerator of Eq. (26) can only be expressed in terms of complex radicals, although the roots are real actually. Still, Eq. (26) can be solved for the limiting cases

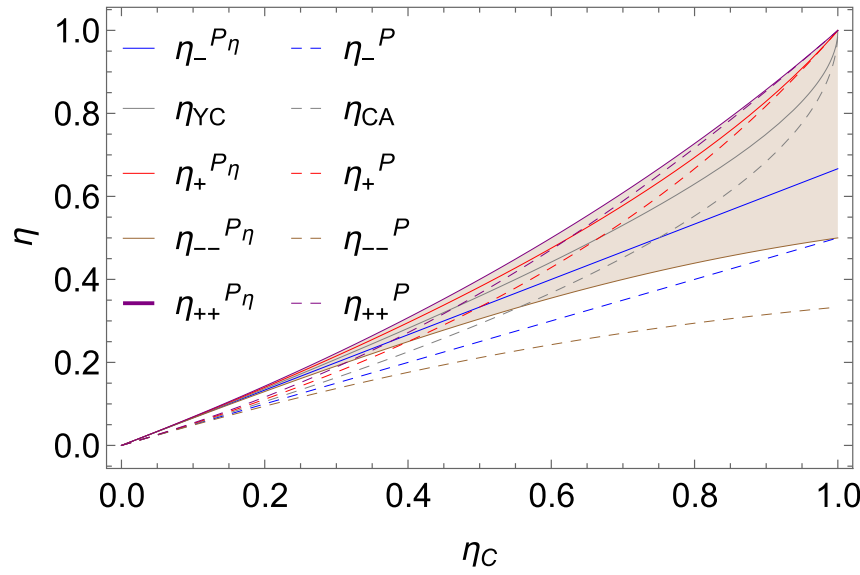

FIG. 3. Efficiency $\eta$ versus the Carnot efficiency $\eta_{C}$ for different operational regimes. The curves under shaded area represent EMEP. Dashed curves represent the corresponding EMP.

$\gamma \rightarrow 0$ and $\gamma \rightarrow \infty$. For $\gamma \rightarrow 0$, the EMEP is evaluated at YC value. For $\gamma \rightarrow \infty$, we obtain $\eta_{+}^{P_{\eta}}=\left(3-\sqrt{9-8 \eta_{C}}\right) / 2$. Hence, EMEP lies in the range

$$
\eta_{Y C} \leqslant \eta_{\omega_{c}}^{P_{\eta}} \leqslant \frac{1}{2}\left(3-\sqrt{9-8 \eta_{C}}\right) \equiv \eta_{+}^{P_{\eta}} .
$$

Upper bound $\eta_{+}^{P_{\eta}}$ obtained here also serves as the upper bound of the low-dissipation model of heat engines $[63,64]$. The same expression also appears in the optimization of Feynman's model operating at MEP in high-temperature regime [69].

The corresponding efficiency bounds for the optimization of the power output of the SSD engine are given by [23]

$$
\begin{gathered}
\eta_{-}^{P} \equiv \frac{\eta_{C}}{2} \leqslant \eta_{\omega_{h}}^{P} \leqslant 1-\sqrt{1-\eta_{C}} \equiv \eta_{C A}, \\
\eta_{C A} \leqslant \eta_{\omega_{c}}^{P} \leqslant \frac{\eta_{C}}{2-\eta_{C}} \equiv \eta_{+}^{P} .
\end{gathered}
$$

Comparing Eqs. (25) and (27) with Eqs. (28) and (29), we can conclude that the SSD engine operating under MEP is far more efficient than the engine operating at MP (see Fig. 3).

\section{B. Weak coupling in the high-temperature regime}

In addition to the strong matter-field coupling regime $\left(\lambda \gg \Gamma_{h, c}\right)$, we can also find analytic expressions for the efficiency in weak matter-field coupling regime $\left(\lambda \ll \Gamma_{h, c}\right)$. In the high-temperature limit $\left(n_{c, h} \gg 1\right)$, the first two terms in the denominator of Eq. (A12) can be ignored. Plus we need extreme dissipation conditions, i.e., either $\Gamma_{c} \gg \Gamma_{h}(\gamma \rightarrow 0)$ or $\Gamma_{c} \ll \Gamma_{h}(\gamma \rightarrow \infty)$. Hence, for $\gamma \rightarrow 0$ and $\gamma \rightarrow \infty$, Eq. (A12) can be approximated by the following two equations, respectively:

$$
\begin{aligned}
& P_{\eta(\gamma \rightarrow 0)}=\frac{2 \hbar \lambda^{2}\left(n_{h}-n_{c}\right)\left(\omega_{h}-\omega_{c}\right)^{2}}{3 \omega_{h} n_{h} n_{c}^{2} \Gamma_{c}}, \\
& P_{\eta(\gamma \rightarrow \infty)}=\frac{\left.2 \hbar \lambda^{2}\left(n_{h}-n_{c}\right) \omega_{h}-\omega_{c}\right)^{2}}{3 \omega_{h} n_{c} n_{h}^{2} \Gamma_{h}} .
\end{aligned}
$$


TABLE I. Taylor series expansions for the various forms of EMEP and EMP obtained under different operational conditions.

\begin{tabular}{lr}
\hline \hline EMEP & EMP \\
\hline$\eta_{--}^{P_{\eta}}=\frac{2}{3} \eta_{C}-\frac{2}{27} \eta_{C}^{2}-\frac{14}{243} \eta_{C}^{3}+O\left(\eta_{C}^{4}\right)$ & $\eta_{--}^{P}=\frac{1}{2} \eta_{C}-\frac{1}{8} \eta_{C}^{2}-\frac{1}{16} \eta_{C}^{3}+O\left(\eta_{C}^{4}\right)$ \\
$\eta_{-}^{P_{\eta}}=\frac{2}{3} \eta_{C}$ & $\eta_{-}^{P}=\frac{1}{2} \eta_{C}$ \\
$\eta_{Y C}=\frac{2}{3} \eta_{C}+\frac{2}{27} \eta_{C}^{2}+\frac{10}{243} \eta_{C}^{3}+O\left(\eta_{C}^{4}\right)$ & $\eta_{C A}=\frac{1}{2} \eta_{C}+\frac{1}{8} \eta_{C}^{2}+\frac{1}{16} \eta_{C}^{3}+O\left(\eta_{C}^{4}\right)$ \\
$\eta_{+}^{P_{\eta}}=\frac{2}{3} \eta_{C}+\frac{4}{27} \eta_{C}^{2}+\frac{16}{243} \eta_{C}^{3}+O\left(\eta_{C}^{4}\right)$ & $\eta_{+}^{P}=\frac{1}{2} \eta_{C}+\frac{2}{8} \eta_{C}^{2}+\frac{2}{16} \eta_{C}^{3}+O\left(\eta_{C}^{4}\right)$ \\
$\eta_{++}^{P_{\eta}}=\frac{2}{3} \eta_{C}+\frac{6}{27} \eta_{C}^{2}+\frac{18}{243} \eta_{C}^{3}+O\left(\eta_{C}^{4}\right)$ & $\eta_{++}^{P}=\frac{1}{2} \eta_{C}+\frac{3}{8} \eta_{C}^{2}+\frac{3}{16} \eta_{C}^{3}+O\left(\eta_{C}^{4}\right)$ \\
\hline \hline
\end{tabular}

Optimization of Eqs. (30) and (31) with respect to $\omega_{c}\left(\omega_{h}\right.$ fixed) yields the following bounds on the efficiency:

$$
\eta_{--}^{P_{\eta}} \equiv \frac{1}{8}\left[3\left(1+\eta_{C}\right)-\sqrt{9 \eta_{C}^{2}-14 \eta_{C}+9}\right] \leqslant \eta_{\omega_{h}}^{P_{\eta}^{\prime}} \leqslant \frac{2 \eta_{C}}{3} .
$$

Note that the above bounds lie below the parametric area bounded by the efficiency curves (area bounded between solid red and blue curves in Fig. 3) given in Eqs. (25) and (27). To the best of our knowledge, these are the new bounds (area bounded between solid brown and solid blue curves in Fig. 3), which are not previously obtained for any model of classical or QHE. Similarly, for the optimization of Eqs. (30) and (31) with respect to $\omega_{h}\left(\omega_{c}\right.$ fixed $)$, EMEP lies in the range

$$
\frac{1}{2}\left(3-\sqrt{9-8 \eta_{C}}\right) \leqslant \eta_{\omega_{h}}^{P_{\eta}^{\prime}} \leqslant \frac{2 \eta_{C}}{3-\eta_{C}} \equiv \eta_{++}^{P_{\eta}} .
$$

Similar to the above case, the area covered (area bounded between solid red and violet curves in Fig. 3) by efficiency curves in Eq. (33) lie above the parametric area bounded by the efficiency curves given in Eq. (27) (see Fig. 3).

The corresponding expressions for the EMP show similar trend (see Fig. 3), and, are given by

$$
\begin{gathered}
\eta_{--}^{P} \equiv \frac{1}{3}\left(1+\eta_{C}-\sqrt{1-\eta_{C}+\eta_{C}^{2}}\right) \leqslant \eta_{\omega_{h}}^{P^{\prime}} \leqslant \frac{\eta_{C}}{2}, \\
\frac{\eta_{C}}{2-\eta_{C}} \leqslant \eta_{\omega_{c}}^{P^{\prime}} \leqslant-1+\eta_{C}+\sqrt{1-\eta_{C}+\eta_{C}^{2}} \equiv \eta_{++}^{P} .
\end{gathered}
$$

Again, the bounds obtained in Eqs. (34) and (35) have not been previously reported for any model of classical or QHE.

We summarize our findings in Table I. As can be seen from Table I, Taylor's series expansions for different expressions for the EMP and EMEP show very interesting behavior. For the EMEP, the first universal term $2 \eta_{C} / 3$ is present in all cases, and the second terms constitute an arithmetic series with common difference $2 \eta_{C}^{2} / 27$. Similarly for the EMP, the first universal term $\eta_{C} / 2$ is present in all cases, and the second term increases by $\eta_{C}^{2} / 8$ in going from the first case to the last case. Additionally, the third terms in the series expansion of various forms of the EMP also constitute an arithmetic series with a common difference $\eta_{C}^{3} / 16$.

\section{Universality of efficiency in one-parameter optimization}

Now, we explore the universal nature of efficiency for oneparameter optimization scheme. We notice that if we put $\Gamma_{c}=$ $\Gamma_{h}(\gamma=1)$, in Eq. (24), the obtained form of the efficiency,

$$
\begin{aligned}
\eta_{\omega_{h}(\gamma=1)}^{P_{\eta}} & =\frac{1}{2}\left(3-\eta_{C}-\sqrt{\left(1-\eta_{C}\right)\left(9-5 \eta_{C}\right)}\right) \\
& =\frac{\eta_{C}}{3}+\frac{\eta_{C}^{2}}{27}+\mathcal{O}\left(\eta_{C}^{3}\right),
\end{aligned}
$$

does not include the second universal term $2 \eta_{C}^{2} / 27$ unlike the case of global optimization over the two parameters as shown in Sec. II. We attribute this to the nature of optimization scheme. The parametric space available to the control variables is different for two different optimization schemes, hence explaining the difference between them. However, one can still retain the second order universal term $2 \eta_{C}^{2} / 27$ if one imposes an extra symmetric condition on the constraints of the optimization. The constraints are symmetric in the sense that under the exchange of the control variables, the constraint equation remains unchanged. The physics of such constraints is explored in Ref. [75]. Here, we want to focus only on the universal character of the efficiency under such constraints. For instance, if we impose a symmetric constrain, viz, $\omega_{c}+\omega_{h}=k$, optimization of Eq. (23) with respect to $\omega_{h}$ leads to the following equation:

$$
\begin{aligned}
x^{3} & +\frac{-(3-7 \tau)}{2(1-\tau)} x^{2}+\frac{-2 \tau(3+4 \tau)}{2(1-\tau)(1+\tau)} x \\
& +\frac{\tau(2+3 \tau)}{2(1-\tau)(1+\tau}=0,
\end{aligned}
$$

where we have put $x=\omega_{c} / k$ for simplicity. The above equation is not solvable in terms of real radicals due to Casus irreducibilis (see Appendix C). However, the equation can be solved in terms of trigonometric functions [76], and the solution is given by

$$
x=-\frac{A}{3}+\frac{2}{3} \sqrt{A^{2}-3 B} \cos \left[\frac{1}{3} \arccos \left(-\frac{2 A^{3}-9 A B+27 C}{2\left(A^{2}-3 B\right)^{3 / 2}}\right)\right],
$$

where $A=-(3-7 \tau) / 2(1-\tau), B=-2 \tau(3+4 \tau) / 2(1-\tau)(1+\tau)$, and $C=\tau(2+3 \tau)$. Substituting above expression in the equation $\eta=1-\omega_{c} / \omega_{h}=(1-2 x) /(1-x)$ (using $\left.\omega_{h}=k-\omega_{c}\right)$, and taking its series expansion, we have

$$
\eta_{k}^{P_{\eta}}=\frac{2}{3} \eta_{C}+\frac{2}{27} \eta_{C}^{2}+\mathcal{O}\left(\eta_{C}^{3}\right)
$$


which clearly shows the presence of the first two universal terms. In a similar manner, for another symmetric constraint $\omega_{c} \omega_{h}=k^{\prime}$, the expression for efficiency, $\eta=1-k^{\prime} / \omega_{h}^{2}$, turns out to be

$$
\begin{aligned}
\eta_{k^{\prime}}^{P_{\eta}} & =1-\frac{3 \tau^{2} M^{1 / 3}}{9 \tau^{4}+36 \tau^{3}+19 \tau^{2}+M[M-\tau(3 \tau+4)]} \\
& =\frac{2}{3} \eta_{C}+\frac{2}{27} \eta_{C}^{2}+\frac{23}{486} \eta_{C}^{3}+\mathcal{O}\left(\eta_{C}^{4}\right),
\end{aligned}
$$

and we again retain the second universal term $2 \eta_{C}^{2} / 27$. Here, $M=\sqrt{k} f(\tau)$, is function of $\tau$ only.

We can also obtain the first two universal terms $\left(\eta_{C} / 2\right.$ and $\left.\eta_{C}^{2} / 8\right)$ in the series expansion of the EMP for the optimization under symmetric constraints. Thus, for the SSD model, we have shown that to establish the universality of efficiency upto the quadratic order term in $\eta_{C}$, we have to impose an additional symmetric condition in addition to the condition $\gamma=1$. Similar is also true for the optimization of an ultrahot Otto engine [75] and Feynman's ratchet model
[77] both of which possess a certain left-right symmetry in the system.

\section{FRACTIONAL LOSS OF POWER AT THE MAXIMUM ECOLOGICAL FUNCTION AND MAXIMUM POWER OUTPUT}

In this section, we make a comparison of the performance of the SSD engine operating at MEP to that of operating at MP. In both cases, we find the expressions for the fractional loss of power due to entropy production, $\dot{S}_{t o t}=\dot{Q}_{c} / T_{c}-\dot{Q}_{h} / T_{h}$. Power loss due to entropy production is given by: $P_{\text {lost }}=$ $T_{2} \dot{S}_{\text {tot }}=\dot{Q}_{c}-\left(1-\eta_{C}\right) \dot{Q}_{h}$. Further using the definitions of power output $P=\dot{Q}_{h}-\dot{Q}_{c}$ and efficiency $\eta=P / \dot{Q}_{h}$, the ratio of power loss to power output can be derived as

$$
R \equiv \frac{P_{\text {lost }}}{P}=\frac{\eta_{C}}{\eta}-1 .
$$

We calculate the ratio $R$ in four different cases: two for the optimization of EP with respect to $\omega_{c}\left(\omega_{h}\right.$ fixed) in the extreme dissipation limits when $\gamma \rightarrow 0$ and $\gamma \rightarrow \infty$, and similar two cases for optimization with respect to $\omega_{h}$ ( $\omega_{c}$ fixed). Using Eqs. (25) and (41), we have

$$
R_{\omega_{h}(\gamma \rightarrow 0)}^{P_{\eta}}=\frac{1}{2}, \quad R_{\omega_{h}(\gamma \rightarrow \infty)}^{P_{\eta}}=\frac{1}{4}\left[\sqrt{\left(9-\eta_{C}\right)\left(1-\eta_{C}\right)}-\left(1-\eta_{C}\right)\right] .
$$

Similar equations for the optimization of $P_{\eta}$ with respect to $\omega_{h}$ for a fixed $\omega_{c}$ can be obtained by using Eqs. (27) and (41):

$$
R_{\omega_{c}(\gamma \rightarrow 0)}^{P_{\eta}}=R_{\omega_{h}(\gamma \rightarrow \infty)}^{P_{\eta}}, \quad R_{\omega_{c}(\gamma \rightarrow \infty)}^{P_{\eta}}=\frac{1}{4}(\sqrt{9-8 c}-1)
$$

For near-equilibrium conditions $\left(\eta_{C} \rightarrow 0\right)$, all the above equations approach the value $1 / 2$ (also see Fig. 4). The fractional loss of power is lower for the case with fixed $\omega_{c}$ than with a fixed $\omega_{h}$. With increasing Carnot efficiency $\eta_{C}$, fractional loss of power decreases, which is natural as, for higher $\eta_{C}$, the engine also operates with higher efficiency wasting less

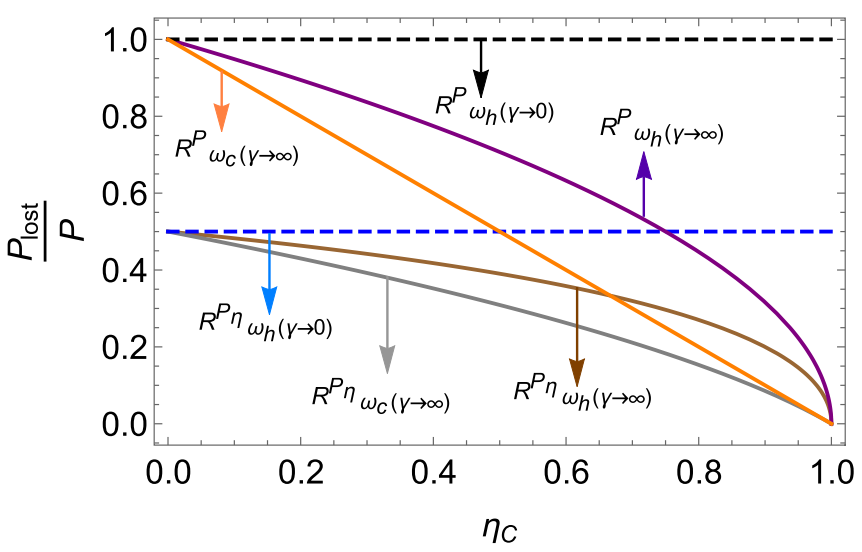

FIG. 4. Comparison of the ratios of power lost to the useful power for two different optimization functions: EP function and power output. The lower-lying curves [Eqs. (42) and (43)] represent the case when EP function is optimized whereas the upper lying curves [Eqs. (44) and (45)] represent the corresponding case for the optimization of power output. fuel. Also, note that $R_{\omega_{c}(\gamma \rightarrow 0)}^{P_{\eta}}=R_{\omega_{h}(\gamma \rightarrow \infty)}^{P_{\eta}}$, as expected, since the corresponding efficiencies are also equal, $\eta_{\omega_{h}(\gamma \rightarrow \infty)}^{P_{\eta}}=$ $\eta_{\omega_{c}(\gamma \rightarrow 0)}^{P_{\eta}}=\eta_{Y C}$, as can be seen from Eqs. (25) and (27). For the SSD engine operating at MP, the ratios of power loss to power output for the different cases discussed above, are given by [25]

$$
\begin{gathered}
R_{\omega_{h}(\gamma \rightarrow 0)}^{P}=1, \quad R_{\omega_{h}(\gamma \rightarrow \infty)}^{P}=\sqrt{1-\eta_{C} .} \\
R_{\omega_{c}(\gamma \rightarrow 0)}^{P}=R_{\omega_{h}(\gamma \rightarrow \infty)}^{P}, \quad R_{\omega_{c}(\gamma \rightarrow \infty)}^{P}=1-\eta_{C} .
\end{gathered}
$$

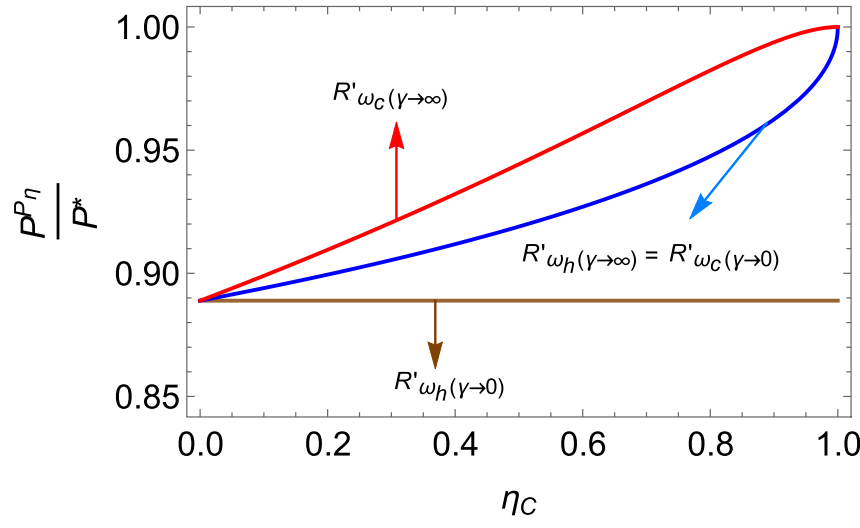

FIG. 5. Comparison of the ratio $R^{\prime}$ of the power output at MEP to the MP [Eqs. (46) and (47)]. 
As can be seen from Fig. 4, the curves representing the optimal power case follow the same trend as noted for the optimal EP. More importantly, for small values of $\eta_{C}$ (nearequilibrium), the curves (lower set) for optimal EP lie well below the curves (upper set) for optimal power. We specifically mention the case where $R_{\omega_{h}(\gamma \rightarrow 0)}^{P}=1$, which implies that in this case, power loss is equal to the power output. The corresponding case for the optimal EP presents us with a much better scenario. In this case, $R_{\omega_{h}(\gamma \rightarrow 0)}^{P_{\eta}}=1 / 2$, which implies that loss of power is half of the power output. It indicates that EP function is a good target function to optimize if our preference is fuel conservation.

\section{RATIO OF POWER AT THE MAXIMUM EFFICIENT POWER TO THE MAXIMUM POWER}

Since the fractional loss of power is less when our the SSD engine operates at MEP as compared to the case when the engine is operating at MP, it is useful to calculate the ratio $\left(R^{\prime}\right)$ of power at MEP to optimal power. Dividing Eq. (B5) by Eq. (B6), and taking the limits $\gamma \rightarrow 0$ and $\gamma \rightarrow \infty$, we get the following two expressions, respectively:

$$
R_{\omega_{h}(\gamma \rightarrow 0)}^{\prime}=\frac{8}{9}, \quad R_{\omega_{h}(\gamma \rightarrow \infty)}^{\prime}=\frac{9-5 \eta_{C}-3 \sqrt{\left(1-\eta_{C}\right)\left(9-\eta_{C}\right)}}{4\left(1-\sqrt{1-\eta_{C}}\right)^{2}} .
$$

Similar equations can be obtained for optimization over $\omega_{h}\left(\right.$ fixed $\left.\omega_{c}\right)$ and are given by

$$
R_{\omega_{c}(\gamma \rightarrow 0)}^{\prime}=R_{\omega_{h}(\gamma \rightarrow \infty)}^{\prime}, \quad R_{\omega_{c}(\gamma \rightarrow 0)}^{\prime}=\frac{\left(3-\sqrt{9-8 \eta_{C}}\right)\left(4 \eta_{C}-3+\sqrt{9-8 \eta_{C}}\right)}{4 \eta_{C}^{2}} .
$$

For very small temperature differences, i.e., $\eta_{C} \rightarrow 0$, the ratio $R^{\prime}=8 / 9$, which shows that at least $88.89 \%$ of the MP is produced in the MEP regime, which is a considerable amount keeping in mind that the power loss in MEP regime is at least $1 / 2$ of the case when the engine operates at MP. It is clear from Fig. 5 that ratio $R^{\prime}$ increases with increasing $\eta_{C}$, which is expected behavior since the efficiency also increases, while the dissipation decreases.

\section{EXPERIMENTAL SETUP OF AN EFFECTIVE THREE-LEVEL HEAT ENGINE}

The three-level model of heat engine can be realized experimentally. In fact, an effective three-level continuous SSD heat engine has already been realized experimentally with nitrogen-vacancy centers in diamond [78]. In the following, we will briefly explain the setup of the experiment conducted.

The working medium of the engine consists of an ensemble of negatively charged nitrogen vacancy centers in diamond. The nitrogen-vacancy center consists of a ground spin-triplet ${ }^{3} A_{2}\left\{|+1\rangle_{g},|0\rangle_{g},|-1\rangle_{g}\right\}$, two excited spin triplets as well as three intermediate singlets $\left({ }^{1} E_{1,2}\right.$ and $\left.{ }^{1} A_{1}\right)$. The degeneracy of the ground and excited states can be lifted by applying a magnetic field. Due to the orbital averaging at room temperature, the two excited state triplets can be treated as a single triplet ${ }^{3} E\left\{|+1\rangle_{e},|0\rangle_{e},|-1\rangle_{e}\right\}\left[\right.$ [78]. Further, the upper $\left({ }^{1} A_{1}\right)$ of the three singlets decays directly into the lower pair ${ }^{1} E$ and has a very short lifetime $(<1 \mathrm{~ns})$, thereby allowing us to treat the three singlet states as an effective single state $\left|0^{\prime}\right\rangle$. By optical excitation [represented by solid brown arrows in Fig. 6(a)], the system is excited to ${ }^{3} E$ triplet. Then the system relaxes back into the ground state triplet $\left\{|+1\rangle_{g},|0\rangle_{g},|-1\rangle_{g}\right\}$ both by spin preserving radiative decay [solid green arrows in Fig. 6(a)] and spin nonpreserving nonradiative decay [diagonal solid blue arrows in Fig. 6(a)] through the meta-stable intermediate state $\left|0^{\prime}\right\rangle$. As an effect of the spin nonpreserving nonradiative decay, the effective rate of population excitation from $|0\rangle_{g}$ to $\left|0^{\prime}\right\rangle$ is much lower than the decay rate from $\left|0^{\prime}\right\rangle$ to $|0\rangle_{g}$, while the effective excitation rate from $|+1\rangle_{g}$ to $\left|0^{\prime}\right\rangle$ is almost equal to its corresponding decay rate [79]. Thus, under the incoherent optical pumping, the $|0\rangle_{g}-\left|0^{\prime}\right\rangle$ and $|+1\rangle_{g}-\left|0^{\prime}\right\rangle$ subsystems can be considered as coupled to the cold and hot baths, respectively. This results in population inversion between the levels $|0\rangle_{g}$ and $|1\rangle_{g}$, which makes the system

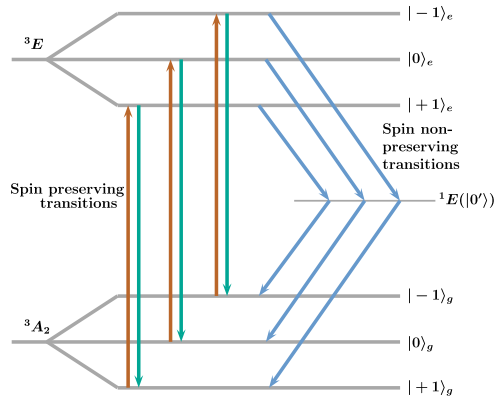

(a)

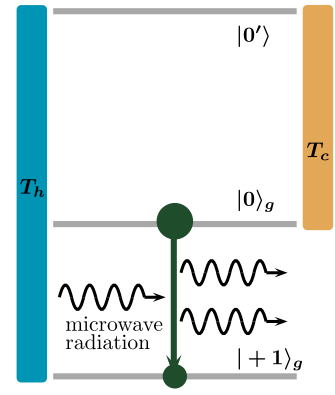

(b)
FIG. 6. Schematics of a three-level quantum heat engine. (a) The relevant energy levels of the nitrogen vacancy center, and spin preserving radiative as well as spin nonpreserving nonradiative transitions between them. (b) The effective three-level heat-engine. The circles represent the steady-state populations. The wavy black arrows represent resonant microwave field and stimulated emission, extracting work.

ready for the lasing action. Then an external microwave field, resonant with the $|+1\rangle_{g} \longleftrightarrow|0\rangle_{g}$ may be applied to extract work in the form of stimulated emission of microwave radiation. Since all microwave transitions involving the level $|-1\rangle_{g}$ are out of resonance, its contribution to the extracted work can be neglected. Hence, the effective system contains only three levels $|1\rangle_{g},|0\rangle_{g}$, and $\left|0^{\prime}\right\rangle$ [see Fig. 6(b)].

The overall working scheme of the effective three-level model explained above is very similar to our model of threelevel SSD engine. In the schematic diagram of the SSD model in Fig. 1, levels $|0\rangle$ and $|1\rangle$ play the role of $|+1\rangle_{g}$ and $|0\rangle_{g}$ (see Fig. 6), respectively. The only difference is that in our model, ground state $|g\rangle$ is connected to both heat reservoirs whereas, in the real experimental setup, meta-stable level $\left|0^{\prime}\right\rangle$ 
plays the same role. However, the overall goal of both schemes remains the same, i.e., to convert incoherent energy of thermal reservoirs into the work by masing (or lasing) action. Moreover, we can also draw the schematics of our SSD model exactly similar to the above-said effective three-level system without affecting the results obtained throughout the paper. Finally, it is worth to mention that besides the SSD engine, an another three-level heat engine, employing $\Lambda$-type atomic system as the working fluid, based on electromagnetically induced transparency has been demonstrated experimentally [80].

\section{CONCLUDING REMARKS}

We have thoroughly investigated the performance of the SSD engine operating under the conditions of MEP and side by side compared its performance with the engine operating at MP. First, for close to the equilibrium conditions, we found an analytic solution for the efficiency of the SSD engine and explicitly showed the universality of the first two terms of both EMP and EMEP under the symmetric dissipation $(\gamma=1)$ condition. Then, we carried out optimization of the EP function alternatively with respect to one of the control frequencies $\omega_{c}$ or $\omega_{h}$ while keeping the other one fixed at a constant value. In the high-temperature limit, we were able to find lower and upper bounds on the EMEP for strong $\left(\lambda \gg \Gamma_{h, c}\right)$ as well as for weak $\left(\lambda \ll \Gamma_{h, c}\right)$ matter-field coupling conditions. Then, we showed that the obtained form of the EMEP in case of one-parameter optimization shows the universal features of efficiency only in the presence of an extra symmetry imposed on the control parameters of the engine. It is important to highlight that except for the weak matter-field coupling $(\lambda \ll$ $\left.\Gamma_{h, c}\right)$ condition, the obtained expressions of the EMEP and EMP in all cases discussed above are the same as obtained for different models of classical heat engine. Specifically, in the weak matter-field coupling regime, we obtained some new bounds on the efficiency of the SSD engine which lie beyond the area covered by bounds obtained for strong matter-field coupling.
Finally, we have compared the optimal performance of the SSD engine operating at MEP to that of operating at MP. It can be inferred that fraction loss of power due to entropy production is appreciably low in the case of heat engine operating at MEP while at the same time it produces at least $88.89 \%$ of the MP output. This indicates that EP function is a good optimization function and real engines should be designed to operate along the lines of maximizing EP function if our preference is environment and fuel conservation. To complete our discussion, we have provided the details of a recent experiment setup in which a three-level heat engine has been demonstrated.

\section{ACKNOWLEDGMENT}

The author gratefully acknowledges useful and fruitful discussions with Kirandeep Kaur.

\section{APPENDIX A: STEADY-STATE SOLUTION OF DENSITY MATRIX EQUATIONS}

Here, we provide steps to solve the equations for density operator in the steady-state. Substituting the expressions for $H_{0}, \bar{H}, V_{0}$, and using Eqs. (2) and (3) in Eq. (4), the time evolution of the matrix elements of the density operator are given by the following equations:

$$
\begin{gathered}
\dot{\rho}_{11}=i \lambda\left(\rho_{10}-\rho_{01}\right)-2 \Gamma_{h}\left[\left(n_{h}+1\right) \rho_{11}-n_{h} \rho_{g g}\right] \\
\dot{\rho}_{00}=-i \lambda\left(\rho_{10}-\rho_{01}\right)-2 \Gamma_{c}\left[\left(n_{c}+1\right) \rho_{00}-n_{c} \rho_{g g}\right] \\
\dot{\rho}_{10}=-\left[\Gamma_{h}\left(n_{h}+1\right)+\Gamma_{c}\left(n_{c}+1\right)\right] \rho_{10}+i \lambda\left(\rho_{11}-\rho_{00}\right) \\
\rho_{11}=1-\rho_{00}-\rho_{g g} \\
\dot{\rho}_{01}=\dot{\rho}_{10}^{*}
\end{gathered}
$$

Solution of the Eqs. (A1)-(A5) in the steady state $\left[\dot{\rho}_{m n}=0\right.$ $(m, n=0,1)]$ yields

$$
\rho_{10}=\frac{i \lambda\left(n_{h}-n_{c}\right) \Gamma_{c} \Gamma_{h}}{\lambda^{2}\left[\left(1+3 n_{h}\right) \Gamma_{h}+\left(1+3 n_{c}\right) \Gamma_{c}\right]+\Gamma_{c} \Gamma_{h}\left[1+2 n_{h}+n_{c}\left(2+3 n_{h}\right)\right]\left[\left(1+n_{c}\right) \Gamma_{c}+\left(1+n_{h}\right) \Gamma_{h}\right]}
$$

and

$$
\rho_{01}=\rho_{10}^{*}
$$

Evaluation of the trace in Eq. (6) leads to the following form of output power,

$$
P=i \hbar \lambda\left(\omega_{h}-\omega_{c}\right)\left(\rho_{01}-\rho_{10}\right)
$$

Similarly calculating the trace in Eq. (5), heat flux $\dot{Q}_{h}$ from the hot reservoir can be written as

$$
\dot{Q}_{h}=-\hbar \omega_{h}\left(2 \Gamma_{h}\left[\left(n_{h}+1\right) \rho_{11}-n_{h} \rho_{g g}\right]\right)
$$

Employing the steady-state condition $\dot{\rho}_{11}=0$ [Eq. (A1)], Eq. (A9) becomes

$$
\dot{Q}_{h}=i \hbar \lambda \omega_{h}\left(\rho_{01}-\rho_{10}\right)
$$


Substituting Eqs. (A6) and (A7) in Eq. (A8), we get the final expression for the power output. Since EP function is just power output multiplied by the efficiency, we have the following expressions for power output and EP, respectively:

$$
\begin{aligned}
P & =\frac{2 \hbar \lambda^{2} \Gamma_{c} \Gamma_{h}\left(n_{h}-n_{c}\right)\left(\omega_{h}-\omega_{c}\right)}{\lambda^{2}\left[\left(1+3 n_{h}\right) \Gamma_{h}+\left(1+3 n_{c}\right) \Gamma_{c}\right]+\Gamma_{c} \Gamma_{h}\left[1+2 n_{h}+n_{c}\left(2+3 n_{h}\right)\right]\left[\left(1+n_{c}\right) \Gamma_{c}+\left(1+n_{h}\right) \Gamma_{h}\right]}, \\
P_{\eta} & =\frac{2 \hbar \lambda^{2} \Gamma_{c} \Gamma_{h}\left(n_{h}-n_{c}\right)\left(\omega_{h}-\omega_{c}\right)^{2}}{\omega_{h} \lambda^{2}\left[\left(1+3 n_{h}\right) \Gamma_{h}+\left(1+3 n_{c}\right) \Gamma_{c}\right]+\Gamma_{c} \Gamma_{h}\left[1+2 n_{h}+n_{c}\left(2+3 n_{h}\right)\right]\left[\left(1+n_{c}\right) \Gamma_{c}+\left(1+n_{h}\right) \Gamma_{h}\right]} .
\end{aligned}
$$

\section{APPENDIX B: OPTIMIZATION OF $P$ AND $P_{\eta}$ IN HIGH-TEMPERATURE AND STRONG-COUPLING REGIME}

In the high-temperatures limit, $n_{h}$ and $n_{c}$ can be approximated as

$n_{h}=\frac{1}{e^{\hbar \omega_{h} / k_{B} T_{h}}-1} \simeq \frac{k_{B} T_{h}}{\hbar \omega_{h}}, \quad n_{c}=\frac{1}{e^{\hbar \omega_{c} / k_{B} T_{c}}-1} \simeq \frac{k_{B} T_{c}}{\hbar \omega_{c}}$.

Using Eq. (B1) in Eq. (A11) and (A12) and dropping the terms containing $\Gamma_{c, h}$ in comparison to $\lambda$, we can write $P$ and $P_{\eta}$ in terms of $\gamma=\Gamma_{h} / \Gamma_{c}$ and $\tau=T_{c} / T_{h}$ in the following form:

$$
\begin{aligned}
P & \simeq \frac{2 \hbar \Gamma_{h}\left(\omega_{h}-\omega_{c}\right)\left(\omega_{c}-\tau \omega_{h}\right)}{3\left(\omega_{c} \gamma+\tau \omega_{h}\right)}, \\
P_{\eta} & \simeq \frac{2 \hbar \Gamma_{h}\left(\omega_{c}-\tau \omega_{h}\right)\left(\omega_{h}-\omega_{c}\right)^{2}}{3 \omega_{h}\left(\omega_{c} \gamma+\tau \omega_{h}\right)} .
\end{aligned}
$$

\section{Expressions for power at MEP and MP a. For fixed $\omega_{h}$}

Optimizing $P_{\eta}$ given in Eq. (B3) with respect to $\omega_{c}$ by setting setting $\partial P / \partial \omega_{c}=0$, we have

$\omega_{c}^{*}=\frac{\gamma \tau \omega_{h}+\sqrt{\gamma+1} \sqrt{\tau} \sqrt{\gamma \tau+8 \gamma+9 \tau} \omega_{h}-3 \tau \omega_{h}}{4 \gamma}$.

Using Eq. (B4) in Eq. (B2), we evaluate the expression for power of the engine operating at MEP:

$$
\begin{aligned}
& P_{\omega_{h}}^{P_{\eta}^{*}} \\
& =\frac{2 \hbar \Gamma_{h} \omega_{h}(\gamma(5 \tau+4)-3 \sqrt{\gamma+1} \sqrt{\tau} \sqrt{(\gamma+9) \tau+8 \gamma}+9 \tau)}{12 \gamma^{2}} .
\end{aligned}
$$

Similarly, the expression for optimal power is given by

$P_{\omega_{h}}^{*}=\frac{2 \hbar \Gamma_{h} \omega_{h}(\gamma+2 \tau+\gamma \tau-2 \sqrt{(\gamma+1) \tau(\gamma+\tau)})}{3 \gamma^{2}}$.

\section{b. For fixed $\omega_{c}$}

Since this case, Casus irreducibilis arises, in order to find the analytic expression for the efficiency, we have to take the limits $\gamma \rightarrow 0$ and $\gamma \rightarrow \infty$ in Eq. (23) before optimizing it. For $\gamma \rightarrow 0$, Eq. (23) is reduced to

$$
P_{\eta(\gamma \rightarrow 0)}=\frac{2 \hbar \Gamma_{h}\left(\omega_{h}-\omega_{c}\right)^{2}\left(\omega_{c}-\tau \omega_{h}\right)}{3 \tau \omega_{c} \omega_{h}} .
$$

Keeping $\omega_{c}$ fixed, and optimizing with respect to $\omega_{h}$, EMEP is evaluated at $\eta_{Y C}$ value. For $\gamma \rightarrow \infty$, Eq. (23) can be written as

$$
P_{\eta(\gamma \rightarrow \infty)}=\frac{2 \hbar \Gamma_{c}\left(\omega_{h}-\omega_{c}\right)^{2}\left(\omega_{c}-\tau \omega_{h}\right)}{3 \omega_{c} \omega_{h}} .
$$

Optimization with respect to $\omega_{h}$ (fixed $\omega_{c}$ ) yields $\eta=$ $\left(3-\sqrt{9-8 \eta_{C}}\right) / 2$.

\section{APPENDIX C: CASUS IRREDUCIBILIS}

While solving a cubic equation, the case of Casus irreducibilis may arise [81,82]. Casus irreducibilis arises when the discriminant $D=18 a b c d-4 b^{3} d+b^{2} c^{2}-4 a c^{3}-$ $27 a^{2} d^{2}$ of a cubic equation

$$
a x^{3}+b x^{2}+c x+d=0 \quad(a, b, c, d \text { are real })
$$

is always positive, i.e., $D>0$. In this case, all three roots of the cubic equation are real. If the roots cannot be found using the rational root test, then the given polynomial is Casus irreducibilis and complex-valued expressions are needed to express the roots in radicals.

In our case, we have to solve the following cubic equation:

$$
\tau^{2} \omega_{h}^{3}+\tau(2 \gamma+\tau) \omega_{c} \omega_{h}^{2}-(\gamma+2 \tau) \omega_{c}^{2} \omega_{h}-\gamma \omega_{c}^{3}=0 .
$$

The discriminant $D$ of the above equation is given by

$$
\begin{aligned}
D= & 4(\gamma+1) \tau^{2}\left(8 \gamma^{3} \tau+\gamma^{3}+12 \gamma^{2} \tau^{2}+6 \gamma^{2} \tau\right. \\
& \left.+6 \gamma \tau^{3}+12 \gamma \tau^{2}+\tau^{4}+8 \tau^{3}\right) \omega_{c}^{6} .
\end{aligned}
$$

Since all the parameters $\omega_{c}, \gamma, \tau$ are positive, $D>0$. So the polynomial in Eq. (C2) presents us with the case of Casus irreducibilis.
[1] H. E. D. Scovil and E. O. Schulz-DuBois, Three-Level Masers as Heat Engines, Phys. Rev. Lett. 2, 262 (1959).

[2] J. E. Geusic, E. O. Schulz-DuBios, and H. E. D. Scovil, Quantum equivalent of the carnot cycle, Phys. Rev. 156, 343 (1967).

[3] J. E. Geusic, E. O. Schulz-DuBois, R. W. De Grasse, and H. E. D. Scovil, Three level spin refrigeration and maser action at $1500 \mathrm{mc} / \mathrm{sec}$, J. Appl. Phys. 30, 1113 (1959).
[4] E. Geva and R. Kosloff, Three-level quantum amplifier as a heat engine: A study in finite-time thermodynamics, Phys. Rev. E 49 3903 (1994).

[5] E. Geva and R. Kosloff, The quantum heat engine and heat pump: An irreversible thermodynamic analysis of the three level amplifier, J. Chem. Phys. 104, 7681 (1996). 
[6] E. Geva, On the irreversible performance of a quantum heat engine, J. Mod. Opt. 49, 635 (2002).

[7] M. O. Scully, Extracting Work from a Single Thermal Bath Via Quantum Negentropy, Phys. Rev. Lett. 87, 220601 (2001).

[8] M. O. Scully, M. S. Zubairy, G. S. Agarwal, and H. Walther, Extracting work from a single heat bath via vanishing quantum coherence, Science 299, 862 (2003).

[9] T. Humphrey and H. Linke, Quantum, cyclic, and particleexchange heat engines, Physica E 29, 390 (2005).

[10] E. Boukobza and D. J. Tannor, Thermodynamics of bipartite systems: Application to light-matter interactions, Phys. Rev. A 74, 063823 (2006).

[11] E. Boukobza and D. J. Tannor, Thermodynamic analysis of quantum light amplification, Phys. Rev. A 74, 063822 (2006).

[12] E. Boukobza and D. J. Tannor, Three-Level Systems as Amplifiers and Attenuators: A Thermodynamic Analysis, Phys. Rev. Lett. 98, 240601 (2007).

[13] M. O. Scully, K. R. Chapin, K. E. Dorfman, M. B. Kim, and A. Svidzinsky, Quantum heat engine power can be increased by noise-induced coherence, Proc. Natl. Acad. Sci. USA 108, 15097 (2011).

[14] U. Harbola, S. Rahav, and S. Mukamel, Quantum heat engines: A thermodynamic analysis of power and efficiency, Europhys. Lett. 99, 50005 (2012).

[15] H. P. Goswami and U. Harbola, Thermodynamics of quantum heat engines, Phys. Rev. A 88, 013842 (2013).

[16] R. Uzdin, A. Levy, and R. Kosloff, Equivalence of Quantum Heat Machines, and Quantum-Thermodynamic Signatures, Phys. Rev. X 5, 031044 (2015).

[17] S. E. Harris, Electromagnetically induced transparency and quantum heat engines, Phys. Rev. A 94, 053859 (2016).

[18] S.-W. Li, M. B. Kim, G. S. Agarwal, and M. O. Scully, Quantum statistics of a single-atom scovil-schulz-dubois heat engine, Phys. Rev. A 96, 063806 (2017).

[19] B. Cleuren, B. Rutten, and C. Van den Broeck, Cooling by Heating: Refrigeration Powered by Photons, Phys. Rev. Lett. 108, 120603 (2012).

[20] J. P. Palao, L. A. Correa, G. Adesso, and D. Alonso, Efficiency of inefficient endoreversible thermal machines, Braz. J. Phys. 46, 282 (2016).

[21] A. Ghosh, C. Latune, L. Davidovich, and G. Kurizki, Catalysis of heat-to-work conversion in quantum machines, Proc. Natl. Acad. Sci. USA 114, 12156 (2017).

[22] A. Ghosh, D. Gelbwaser-Klimovsky, W. Niedenzu, A. I. Lvovsky, I. Mazets, M. O. Scully, and G. Kurizki, Two-level masers as heat-to-work converters, Proc. Natl. Acad. Sci. USA 115, 9941 (2018).

[23] K. E. Dorfman, D. Xu, and J. Cao, Efficiency at maximum power of a laser quantum heat engine enhanced by noiseinduced coherence, Phys. Rev. E 97, 042120 (2018).

[24] C. Karg1, M. T. Naseem, T. Opatrn, Ö. E. Müstecaplıŏlu, and G. Kurizki, Quantum optical two-atom thermal diode, Phys. Rev. E 99, 042121 (2019).

[25] V. Singh and R. S. Johal, Three-level laser heat engine at optimal performance with ecological function, Phys. Rev. E 100, 012138 (2019).

[26] V. Singh, T. Pandit, and R. S. Johal, Optimal performance of a three-level quantum refrigerator, Phys. Rev. E 101, 062121 (2020).
[27] P. Menczel, C. Flindt, and K. Brandner, Thermodynamics of cyclic quantum amplifiers, Phys. Rev. A 101, 052106 (2020).

[28] N. Linden, S. Popescu, and P. Skrzypczyk, How Small Can Thermal Machines Be? The Smallest Possible Refrigerator, Phys. Rev. Lett. 105, 130401 (2010).

[29] A. Levy and R. Kosloff, Quantum Absorption Refrigerator, Phys. Rev. Lett. 108, 070604 (2012).

[30] L. A. Correa, J. P. Palao, G. Adesso, and D. Alonso, Performance bound for quantum absorption refrigerators, Phys. Rev. E 87, 042131 (2013).

[31] B. K. Agarwalla, J.-H. Jiang, and D. Segal, Quantum efficiency bound for continuous heat engines coupled to noncanonical reservoirs, Phys. Rev. B 96, 104304 (2017).

[32] M. Kilgour and D. Segal, Coherence and decoherence in quantum absorption refrigerators, Phys. Rev. E 98, 012117 (2018).

[33] G. Maslennikov, S. Ding, R. Hablützel, J. Gan, A. Roulet, S. Nimmrichter, J. Dai, V. Scarani, and D. Matsukevich, Quantum absorption refrigerator with trapped ions, Nat. Commun. 10, 202 (2019).

[34] M. T. Mitchison, M. Huber, J. Prior, M. P. Woods, and M. B. Plenio, Realising a quantum absorption refrigerator with an atom-cavity system, Quantum Sci. Technol. 1, 015001 (2016).

[35] J. B. Brask and N. Brunner, Small quantum absorption refrigerator in the transient regime: Time scales, enhanced cooling, and entanglement, Phys. Rev. E 92, 062101 (2015).

[36] M. T. Naseem, A. Misra, and Ö. E. Müstecaplığlu, Two-body quantum absorption refrigerators with optomechanical-like interactions, Quantum Sci. Technol. 5, 035006 (2020).

[37] R. Alicki, The quantum open system as a model of the heat engine, J. Phys. A 12, L103 (1979).

[38] N. Jaseem, M. Hajdušek, V. Vedral, R. Fazio, L.-C. Kwek, and S. Vinjanampathy, Quantum synchronization in nanoscale heat engines, Phys. Rev. E 101, 020201(R) (2020).

[39] R. Kosloff, A quantum mechanical open system as a model of a heat engine, J. Chem. Phys. 80, 1625 (1984).

[40] E. Geva and R. Kosloff, On the classical limit of quantum thermodynamics in finite time, J. Chem. Phys. 97, 4398 (1992).

[41] E. Geva and R. Kosloff, A quantum mechanical heat engine operating in finite time. a model consisting of spin-1/2 systems as the working fluid, J. Chem. Phys. 96, 3054 (1992).

[42] B. Lin and J. Chen, Performance analysis of an irreversible quantum heat engine working with harmonic oscillators, Phys. Rev. E 67, 046105 (2003).

[43] O. Abah, J. Roßnagel, G. Jacob, S. Deffner, F. Schmidt-Kaler, K. Singer, and E. Lutz, Single-Ion Heat Engine at Maximum Power, Phys. Rev. Lett. 109, 203006 (2012).

[44] S. Deffner, Efficiency of harmonic quantum otto engines at maximal power, Entropy 20, 875 (2018).

[45] B. Andresen, P. Salamon, and R. S. Berry, Thermodynamics in finite time, Phys. Today 37(9), 62 (1984).

[46] P. Salamon, J. Nulton, G. Siragusa, T. Andersen, and A. Limon, Principles of control thermodynamics, Energy 26, 307 (2001).

[47] B. Andresen, Current trends in finite-time thermodynamics, Angew. Chem. Int. Ed. 50, 2690 (2011).

[48] F. L. Curzon and B. Ahlborn, Efficiency of a Carnot engine at maximum power output, Am. J. Phys. 43, 22 (1975).

[49] M. H. Rubin, Optimal configuration of a class of irreversible heat engines. I, Phys. Rev. A 19, 1272 (1979). 
[50] M. Esposito, R. Kawai, K. Lindenberg, and C. Van den Broeck, Efficiency at Maximum Power of Low-Dissipation Carnot Engines, Phys. Rev. Lett. 105, 150603 (2010).

[51] M. Esposito, R. Kawai, K. Lindenberg, and C. Van den Broeck, Quantum-dot carnot engine at maximum power, Phys. Rev. E 81, 041106 (2010).

[52] V. Cavina, A. Mari, and V. Giovannetti, Slow Dynamics and Thermodynamics of Open Quantum Systems, Phys. Rev. Lett. 119, 050601 (2017).

[53] M. Esposito, K. Lindenberg, and C. Van den Broeck, Universality of Efficiency at Maximum Power, Phys. Rev. Lett. 102, 130602 (2009).

[54] C. Van den Broeck, Thermodynamic Efficiency at Maximum Power, Phys. Rev. Lett. 95, 190602 (2005).

[55] A. C. Hernández, A. Medina, J. M. M. Roco, J. A. White, and S. Velasco, Unified optimization criterion for energy converters, Phys. Rev. E 63, 037102 (2001).

[56] F. Angulo-Brown, An ecological optimization criterion for finite time heat engines, J. Appl. Phys. 69, 7465 (1991).

[57] J. W. Stucki, The optimal efficiency and the economic degrees of coupling of oxidative phosphorylation, Eur. J. Biochem. 109, 269 (1980).

[58] Z. Yan and J. Chen, A generalized rutgers formula derived from the theory of endoreversible cycles, Phys. Lett. A 217, 137 (1996).

[59] Y. Zhang, C. Huang, G. Lin, and J. Chen, Universality of efficiency at unified trade-off optimization, Phys. Rev. E 93, 032152 (2016).

[60] Y. Zhang, J. Guo, G. Lin, and J. Chen, Universal optimization efficiency for nonlinear irreversible heat engines, J. NonEquilib. Thermodyn. 42, 253 (2017).

[61] A. de Vos, Endoreversible Thermodynamics of Solar Energy Conversion (Oxford University Press, Oxford, UK, 1992).

[62] J. Chen, Z. Yan, G. Lin, and B. Andresen, On the curzonahlborn efficiency and its connection with the efficiencies of real heat engines, Energy Convers. and Manage. 42, 173 (2001).

[63] V. Singh and R. S. Johal, Low-dissipation carnot-like heat engines at maximum efficient power, Phys. Rev. E 98, 062132 (2018).

[64] V. Holubec and A. Ryabov, Efficiency at and near maximum power of low-dissipation heat engines, Phys. Rev. E 92, 052125 (2015).

[65] L. Chen, Z. Ding, J. Zhou, W. Wang, and F. Sun, Thermodynamic performance optimization for an irreversible vacuum thermionic generator, Eur. Phys. J. Plus 132, 293 (2017).

[66] L. A. Arias-Hernandez, F. Angulo-Brown, and R. T. PaezHernandez, First-order irreversible thermodynamic approach to a simple energy converter, Phys. Rev. E 77, 011123 (2008).
[67] J. Chimal-Eguia, R. Paez-Hernandez, D. Ladino-Luna, and J. M. Velázquez-Arcos, Performance of a simple energeticconverting reaction model using linear irreversible thermodynamics, Entropy 21, 1030 (2019).

[68] N. Sánchez-Salas, J. Chimal-Eguía, and M. Ramírez-Moreno, Optimum performance for energy transfer in a chemical reaction system, Physica A 446, 224 (2016).

[69] V. Singh and R. S. Johal, Performance of Feynman's ratchet under a trade-off figure of merit: Exact analysis versus estimation from prior information, J. Stat. Mech. (2019) 093208.

[70] N. M. Myers and S. Deffner, Bosons outperform fermions: The thermodynamic advantage of symmetry, Phys. Rev. E 101, 012110 (2020).

[71] T. Yilmaz, A new performance criterion for heat engines: Efficient power, J. Energy Inst. 79, 38 (2006).

[72] R. P. Feynman, R. B. Leighton, and M. Sands, The Feynman Lectures on Physics (Narosa Publishing House, New Delhi, India, 2008).

[73] Z. C. Tu, Efficiency at maximum power of Feynman's ratchet as a heat engine, J. Phys. A: Math. Theor. 41, 312003 (2008).

[74] V. Singh and R. S. Johal, Feynman's ratchet and pawl with ecological criterion: Optimal performance versus estimation with prior information, Entropy 19, 576 (2017).

[75] R. Uzdin and R. Kosloff, Universal features in the efficiency at maximal work of hot quantum otto engines, Europhys Lett. 108, 40001 (2014).

[76] P. M. Radmore and S. M. Barnett, Methods in Theoretical Quantum Optics (Oxford University Press, Oxford, 1997).

[77] V. Singh and R. S. Johal, Feynman-smoluchowski engine at high temperatures and the role of constraints, J. Stat. Mech. (2018) 073205.

[78] J. Klatzow, J. N. Becker, P. M. Ledingham, C. Weinzetl, K. T. Kaczmarek, D. J. Saunders, J. Nunn, I. A. Walmsley, R. Uzdin, and E. Poem, Experimental Demonstration of Quantum Effects in the Operation of Microscopic Heat Engines, Phys. Rev. Lett. 122, 110601 (2019).

[79] J. Tetienne, L. Rondin, P. Spinicelli, M. Chipaux, T. Debuisschert, J. Roch, and V. Jacques, Magnetic-fielddependent photodynamics of single nv defects in diamond: an application to qualitative all-optical magnetic imaging, New J. Phys. 14, 103033 (2012).

[80] Y. Zou, Y. Jiang, Y. Mei, X. Guo, and S. Du, Quantum Heat Engine using Electromagnetically Induced Transparency, Phys. Rev. Lett. 119, 050602 (2017).

[81] I. Kleiner, Biographies of selected mathematicians, in A History of Abstract Algebra (Birkhäuser, Boston, 2007), pp. 113-163.

[82] I. Stewart, Galois Theory (Chapman and Hall, CRC, New York, 1990). 